\title{
Construction of Unique Structure of Ru Sites in RuP Structure for Propane Dehydrogenation
}

\author{
Tianxing Yang $\stackrel{a, b \#}{ }$, Yuan Zhong $\stackrel{a \#}{\underline{a \#}}$, Jiale Li $i^{a}$, Rui Ma ${ }^{\underline{c}}$, Hong Yan $\underline{a}^{*}$, Yanan Liu $\stackrel{a, b}{ }$, Yufei He $\stackrel{a, b}{ }^{*}$, \\ Dianqing $\operatorname{Li}^{a, b^{*}}$ \\ ${ }^{a}$ State Key Laboratory of Chemical Resource Engineering, Beijing University of Chemical Technology, Beijing \\ 100029, China \\ beijing Engineering Center for Hierarchical Catalysts, Beijing University of Chemical Technology, Beijing \\ 100029, China \\ ${ }^{\complement}$ Chemistry and Chemical Engineering Guangdong Laboratory, Shantou, 515031, P.R. China
}

* Corresponding author

Address: Box 98, 15 Bei San Huan East Road, Beijing 100029, China

Tel: $+861064436992 \quad$ Fax: +861064436992

E-mail address: yanhong@mail.buct.edu.cn (Hong Yan); yfhe@mail.buct.edu.cn (Yufei He); $\underline{\text { lidq@,mail.buct.edu.cn (Dianqing Li) }}$ 


\section{The calculation of the surface energy.}

Surface energy (SE) is defined as the amount of energy required to cleave an infinite crystal into two parts, i.e. the energy required to form a new surface. It is calculated as shown in eqs1:

$$
E_{\text {surf }}=\frac{1}{2 A}\left(E_{\text {slab }}-E_{\text {bulk }}\right)
$$

Here, $E_{\text {slab }}$ is the total energy of the slab, $E_{\text {bulk }}$ is the energy in the bulk. In general, it is known that the smaller the surface energy is, the easier is to form a surface, i.e., the surface with smaller surface energy is easier to be exposed.
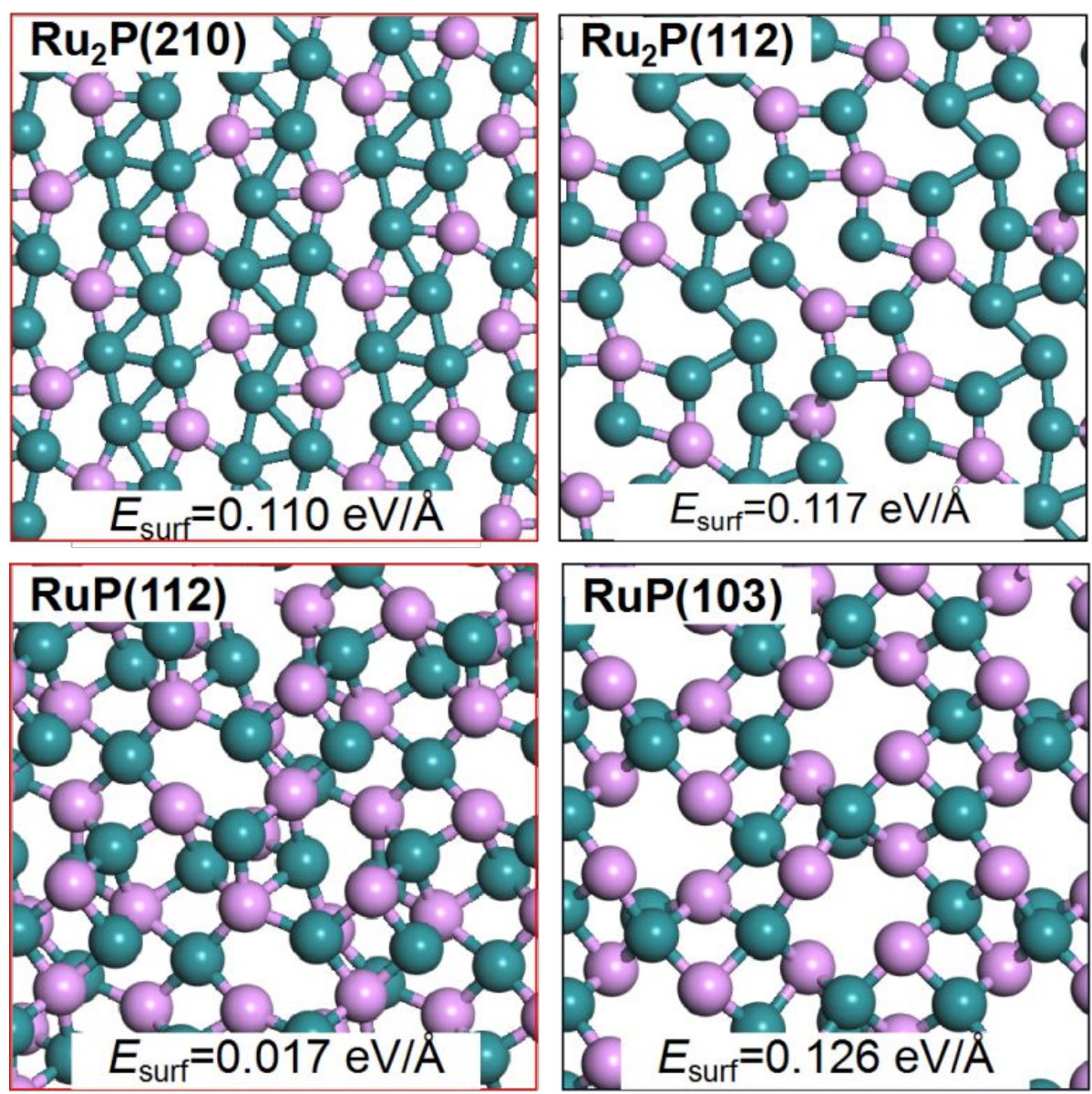

Figure $\mathrm{S} 1$. The top view of the optimized structures of $\mathrm{Ru}_{2} \mathrm{P}(210), \mathrm{Ru}_{2} \mathrm{P}(112), \mathrm{RuP}(112)$ and RuP(103). Ru:cyan; P:purple. 


\section{Energy test for K-point.}

As shown in Table S1, when the K-point is set to $3 \times 3 \times 1$, the adsorption energy is $-0.565 \mathrm{eV}$, and when the K-point is increased to $5 \times 5 \times 1$, the DFT calculation results show that the adsorption energy has only a small change $(0.001 \mathrm{eV})$. Therefore, in order to save computing resources, the K-point of $3 \times 3 \times 1$ is used to complete all calculations.

Table S1. Adsorption energy of methanol molecules on RuP(112) surface when K-point are $3 \times 3 \times 1$ and $5 \times 5 \times 1($ Unit: $\mathrm{eV})$

\begin{tabular}{lllll}
\hline K-point & $E_{\text {surface }}$ & $E_{\text {adsorbate }}$ & $E_{\text {adsorbate/surface }}$ & $E_{\text {ads }}$ \\
\hline $3 \times 3 \times 1$ & -229.549 & -57.012 & -287.126 & -0.565 \\
$5 \times 5 \times 1$ & -229.549 & -57.012 & -287.127 & -0.566 \\
\hline
\end{tabular}

\section{Energy test for cut-off energy (ENCUT).}

In order to make reasonable use of computing resources, we tested the cutoff energy (ENCUT) required for the calculation. If the ENCUT value is too small, the system will be difficult to converge, and if it is too large, it will take longer to waste computing resources. As shown in Figure S2, for the system we want to study, when the ENCUT value is $450 \mathrm{eV}$, it can not only ensure the convergence of the system, but also save computing resources. 


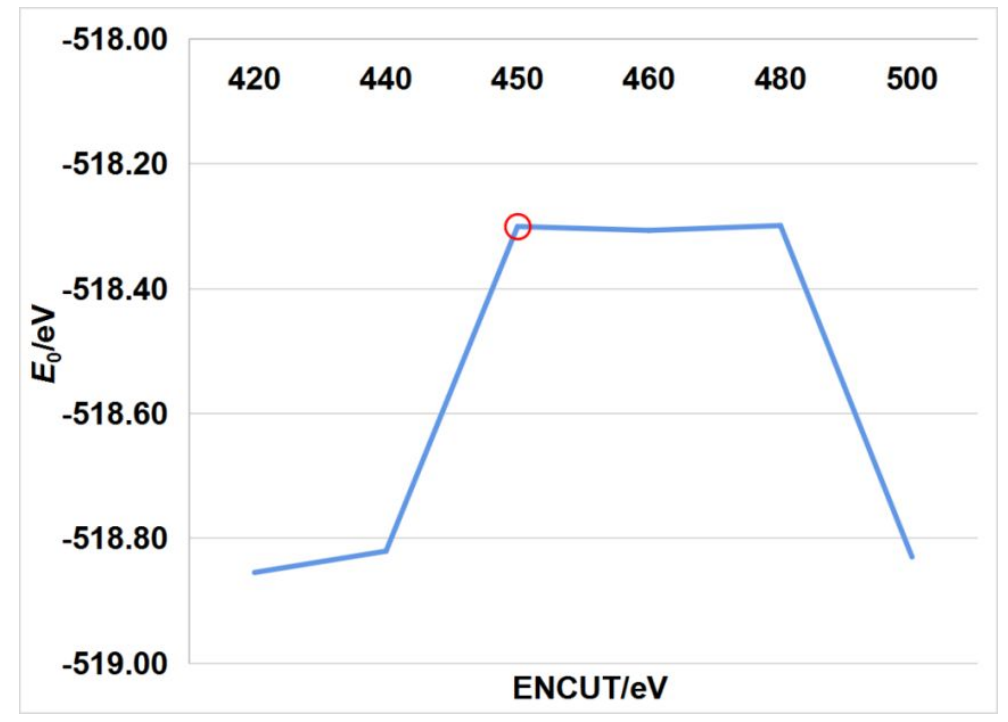

Figure S2. Take the energy $E_{0}$ of $\mathrm{CH}_{3} \mathrm{OH}$ adsorbed on $\mathrm{RuP}(112)$ surface as an example for ENCUT test.

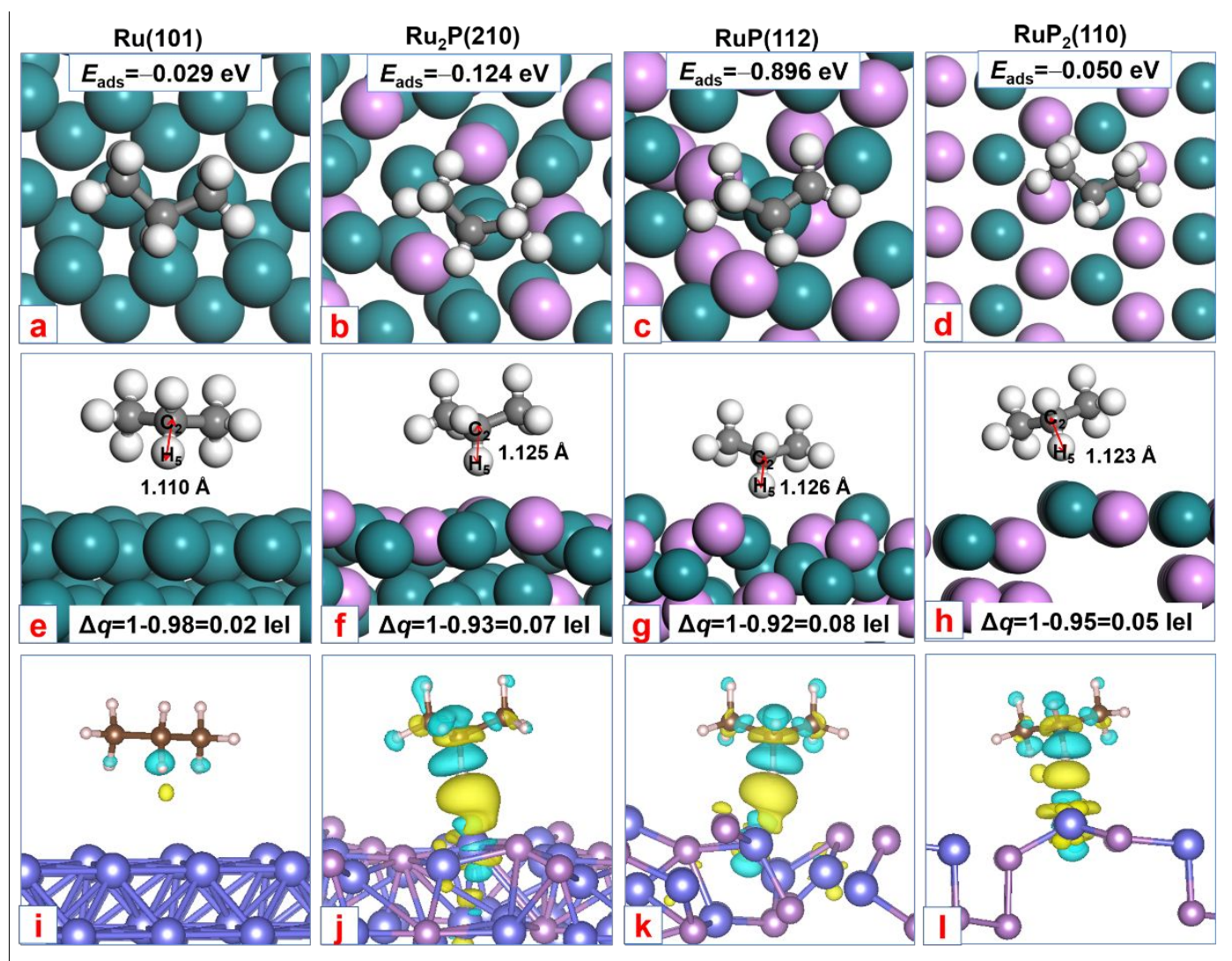

Figure S3. The top (a-d) and side (e-h) view of adsorption structures of propane on $\mathrm{Ru}$ and $\mathrm{Ru}_{x} \mathrm{P}_{y}$ crystal surfaces, along with their adsorption energies $\left(E_{\text {ads }}\right)$ and charge density difference analyses (i-1). The distance in the figure represents the $\mathrm{C}_{2}-\mathrm{H}_{5}$ bond length, and the $\Delta q$ represents the charge transfer of the $\mathrm{H}_{5}$ atom before and after adsorption, $\mathrm{Ru}$ : cyan; $\mathrm{P}$ : purple; C: black; $\mathrm{H}$ : white. 


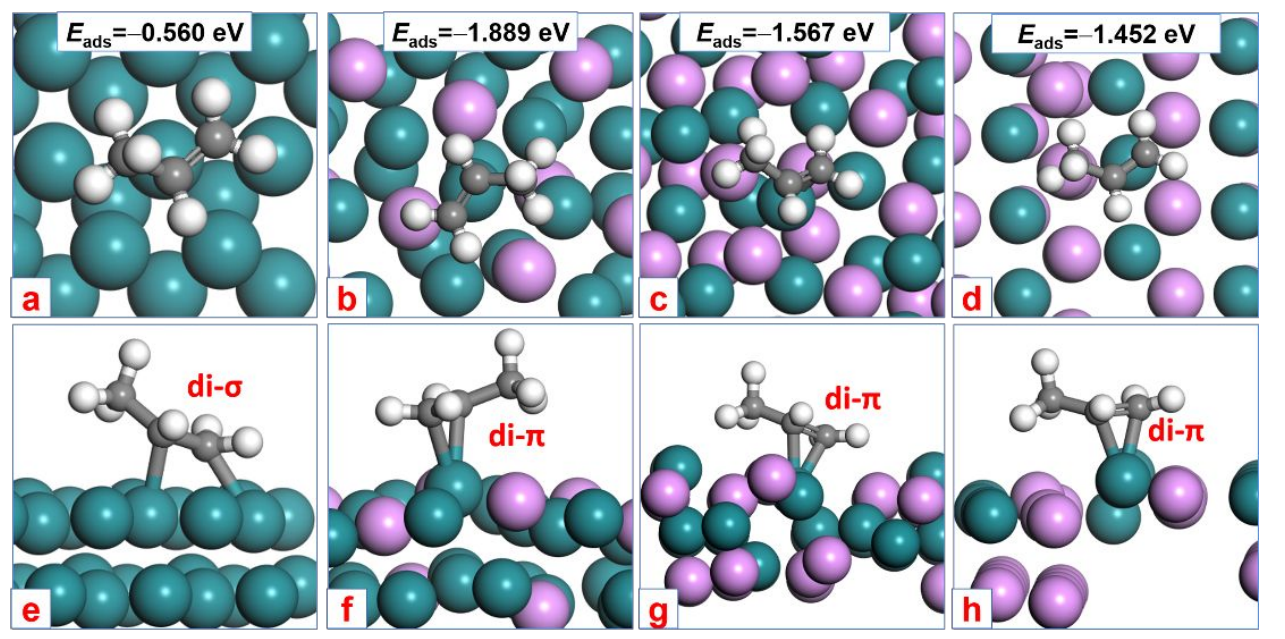

Figure S4. The top (a-d) and side (e-h) view of adsorption structures of propylene on Ru and $\mathrm{Ru}_{x} \mathrm{P}_{y}$ crystal surfaces, Ru: cyan; P: purple; C: black; H: white.

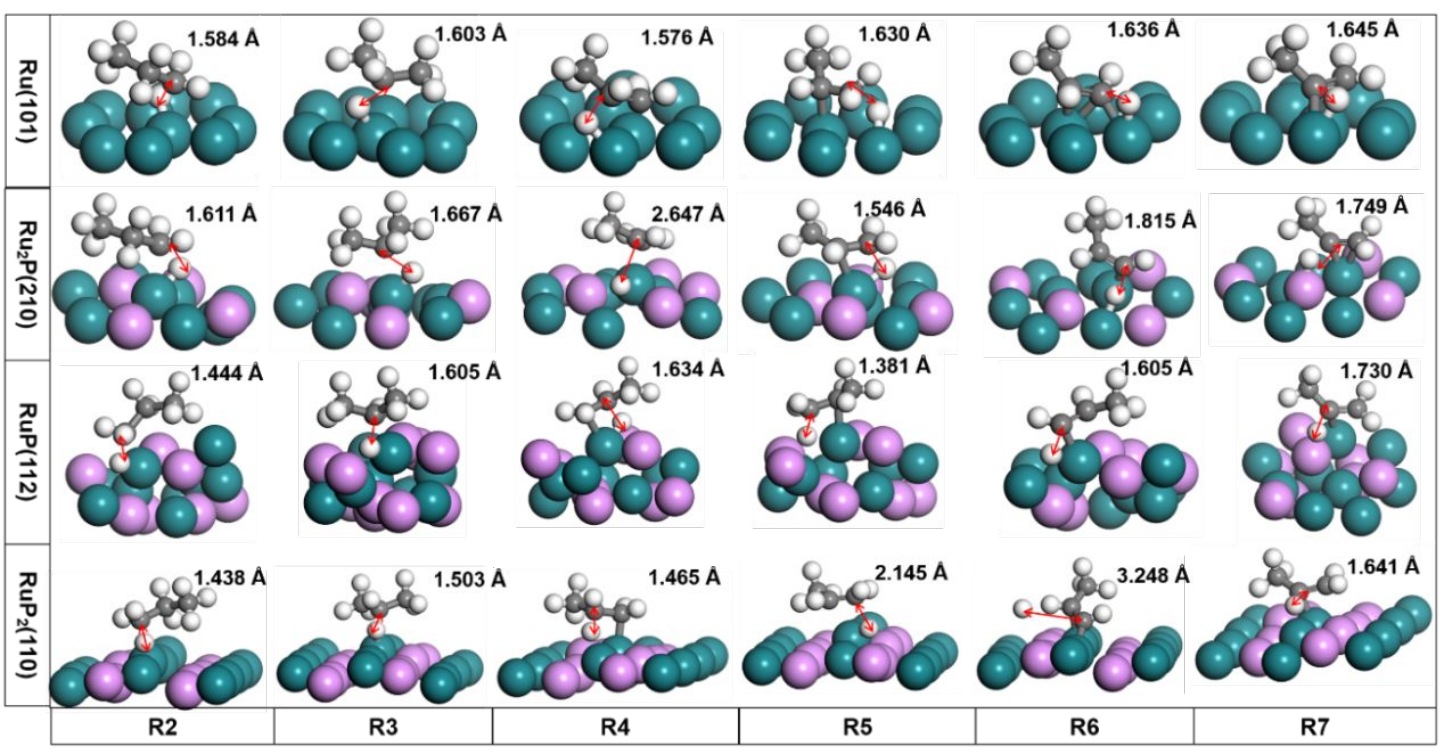

Figure S5. The side view of the transition state structure of propane dehydrogenation, where the lengths of the activated $\mathrm{C}-\mathrm{H}$ bonds are also given, Ru:cyan; P:purple; C:black; H:white. 
Table S2. Adsorption energies of propane and other reaction intermediates on $\mathrm{Ru}$ and $\mathrm{Ru}_{x} \mathrm{P}_{y}$ surfaces (unit: eV)

\begin{tabular}{lllllll}
\hline & $\mathrm{CH}_{3} \mathrm{CH}_{2} \mathrm{CH}_{3}$ & $\mathrm{CH}_{3} \mathrm{CHCH}_{3}$ & $\mathrm{CH}_{3} \mathrm{CH}_{2} \mathrm{CH}_{2}$ & $\mathrm{CH}_{3} \mathrm{CHCH}_{2}$ & $\mathrm{CH}_{3} \mathrm{CHCH}$ & $\mathrm{CH}_{3} \mathrm{CCH}_{2}$ \\
\hline $\mathrm{Ru}$ & -0.029 & -1.005 & -1.683 & -0.560 & -3.026 & -2.617 \\
$\mathrm{Ru}_{2} \mathrm{P}$ & -0.124 & -3.939 & -2.506 & -1.889 & -3.229 & -3.424 \\
$\mathrm{RuP}$ & -0.896 & -2.126 & -2.614 & -1.567 & -2.803 & -2.799 \\
$\mathrm{RuP}_{2}$ & -0.050 & -1.868 & -2.476 & -1.452 & -3.648 & -2.517 \\
\hline
\end{tabular}

Table S3. The surface roughness $(R)$ of $\mathrm{Ru}_{x} \mathrm{P}_{y}$ surfaces

\begin{tabular}{ccccc}
\hline & $\mathrm{Ru}(101)$ & $\mathrm{Ru}_{2} \mathrm{P}(210)$ & $\mathrm{RuP}(112)$ & $\mathrm{RuP}_{2}(110)$ \\
\hline$R / 10^{-3} \AA$ & 7.75 & 15.49 & 37.55 & 21.21 \\
\hline
\end{tabular}

Table S4. Dehydrogenation reaction energy barriers of propane on $\mathrm{Ru}$ and $\mathrm{Ru}_{x} \mathrm{P}_{y}$ surfaces (unit: $\mathrm{eV}$ )

\begin{tabular}{|c|c|c|c|c|c|}
\hline & Reaction & $\mathrm{Ru}$ & $\mathrm{Ru}_{2} \mathrm{P}$ & RuP & $\mathrm{RuP}_{2}$ \\
\hline $\mathrm{R} 1$ & $\mathrm{CH}_{3} \mathrm{CH}_{2} \mathrm{CH}_{3}(\mathrm{~g}) \rightarrow \mathrm{CH}_{3} \mathrm{CH}_{2} \mathrm{CH}_{3} *$ & -- & -- & -- & -- \\
\hline $\mathrm{R} 2$ & $\mathrm{CH}_{3} \mathrm{CH}_{2} \mathrm{CH}_{3} * \rightarrow \mathrm{CH}_{3} \mathrm{CH}_{2} \mathrm{CH}_{2} *+\mathrm{H}^{*}$ & 0.949 & 0.947 & 0.833 & 0.636 \\
\hline R3 & $\mathrm{CH}_{3} \mathrm{CH}_{2} \mathrm{CH}_{3} * \rightarrow \mathrm{CH}_{3} \mathrm{CHCH}_{3} *+\mathrm{H}^{*}$ & 1.156 & 0.934 & 0.714 & 0.790 \\
\hline R4 & $\mathrm{CH}_{3} \mathrm{CH}_{2} \mathrm{CH}_{2}{ }^{*} \rightarrow \mathrm{CH}_{3} \mathrm{CHCH}_{2} *+\mathrm{H}^{*}$ & 0.373 & 0.811 & 0.870 & 0.414 \\
\hline R5 & $\mathrm{CH}_{3} \mathrm{CHCH}_{3} * \rightarrow \mathrm{CH}_{3} \mathrm{CHCH}_{2} *+\mathrm{H}^{*}$ & 0.645 & 0.866 & 0.649 & 0.910 \\
\hline R6 & $\mathrm{CH}_{3} \mathrm{CHCH}_{2} * \rightarrow \mathrm{CH}_{3} \mathrm{CHCH}^{*}+\mathrm{H}^{*}$ & 0.320 & 1.913 & 1.796 & 2.820 \\
\hline R7 & $\mathrm{CH}_{3} \mathrm{CHCH}_{2} * \rightarrow \mathrm{CH}_{3} \mathrm{CCH}_{2} *+\mathrm{H}^{*}$ & 0.706 & 1.554 & 2.022 & 1.037 \\
\hline
\end{tabular}


Table S5. Energy barrier difference between further dehydrogenation and desorption of propylene on $\mathrm{Ru}$ and $\mathrm{Ru}_{x} \mathrm{P}_{y}$ surfaces (unit: $\mathrm{eV}$ )

\begin{tabular}{cccccc}
\hline & & $\mathrm{Ru}(101)$ & $\mathrm{Ru}_{2} \mathrm{P}(210)$ & $\mathrm{RuP}(112)$ & $\mathrm{RuP}_{2}(110)$ \\
\hline $\mathrm{R} 6$ & $E_{\text {adesorption }}$ & 0.560 & 1.889 & 1.567 & 1.452 \\
$\mathrm{CH}_{3} \mathrm{CHCH}_{2}{ }^{*} \rightarrow$ & $E_{\text {adehydrogenation }}$ & 0.320 & 1.913 & 1.796 & 2.820 \\
$\mathrm{CH}_{3} \mathrm{CHCH}^{*}+\mathrm{H}^{*}$ & $E_{\text {diff }}$ & -0.240 & 0.024 & 0.229 & 1.368 \\
$\mathrm{R} 7$ & $E_{\text {adesorption }}$ & 0.560 & 1.889 & 1.567 & 1.452 \\
$\mathrm{CH}_{3} \mathrm{CHCH}_{2} * \rightarrow$ & $E_{\text {adehydrogenation }}$ & 0.706 & 1.554 & 2.022 & 1.037 \\
$\mathrm{CH}_{3} \mathrm{CCH}_{2} *+\mathrm{H}^{*}$ & $E_{\text {diff }}$ & 0.147 & -0.335 & 0.455 & -0.414 \\
\hline
\end{tabular}

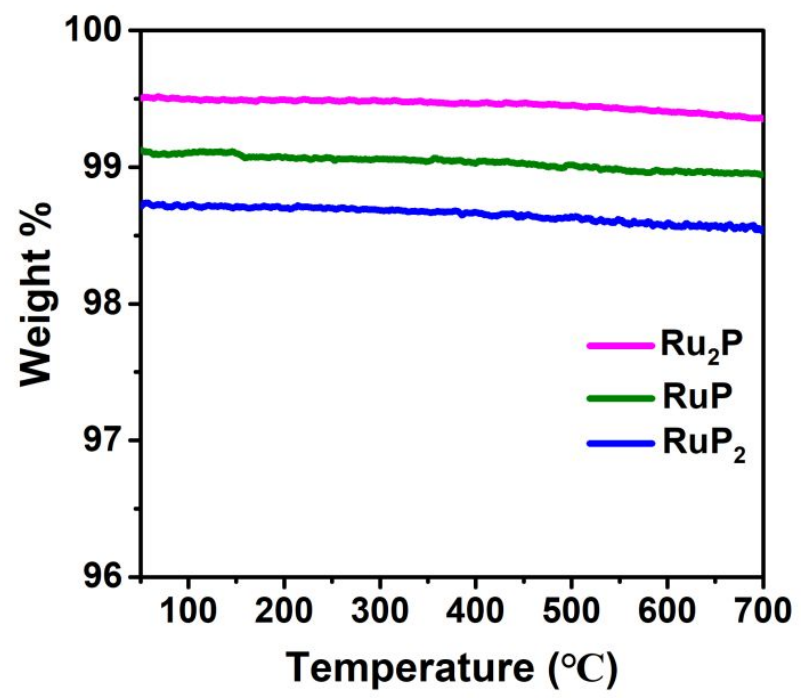

Figure S6. TG curves under $\mathrm{N}_{2}$ atmosphere of fresh $\mathrm{Ru}_{x} \mathrm{P}_{y}$ samples.

Table S6. BET data and atomic ratio of $\mathrm{Ru}_{x} \mathrm{P}_{y}$ catalysts by EDX

\begin{tabular}{ccccc}
\hline Samples & $\mathbf{S}_{\mathbf{B E T}}\left(\mathbf{m}^{\mathbf{2}} \mathbf{g}^{-\mathbf{1}}\right)$ & $\begin{array}{c}\text { Pore volume } \\
\left(\mathbf{c m}^{\mathbf{3}} \mathbf{g}^{-\mathbf{1}}\right)\end{array}$ & $\begin{array}{c}\text { Average pore } \\
\text { diameter } \mathbf{( n m})\end{array}$ & P/Ru atomic ratio \\
\hline $\mathrm{Ru}_{2} \mathrm{P}$ & 31 & 0.07 & 9.2 & 0.47 \\
$\mathrm{RuP}$ & 24 & 0.07 & 11.1 & 1.05 \\
$\mathrm{RuP}_{2}$ & 20 & 0.03 & 6.7 & 1.93 \\
\hline
\end{tabular}


Table S7. PDH catalytic performance (180 min) of four samples

\begin{tabular}{ccccccc}
\hline \multirow{2}{*}{ Catalyst } & \multicolumn{2}{c}{ Conversion $\%$} & Propylene selectivity $\%$ & \multicolumn{2}{c}{ Carbon balance $\%$} \\
\cline { 2 - 6 } & Initial & Steady-state & Initial & Steady-state & Initial & Steady-state \\
\hline $\mathrm{Ru} / \mathrm{SiO}_{2}$ & 16.9 & 9.8 & 13.2 & 59.8 & $73 \pm 5$ & $95 \pm 5$ \\
$\mathrm{Ru}_{2} \mathrm{P}$ & 19.8 & 11.4 & 55.3 & 72.2 & $80 \pm 5$ & $97 \pm 5$ \\
$\mathrm{RuP}$ & 18.8 & 12.7 & 87.2 & 90.5 & $90 \pm 5$ & $98 \pm 5$ \\
$\mathrm{RuP}_{2}$ & 19.1 & 11.2 & 42.3 & 67.7 & $83 \pm 5$ & $97 \pm 5$ \\
\hline
\end{tabular}
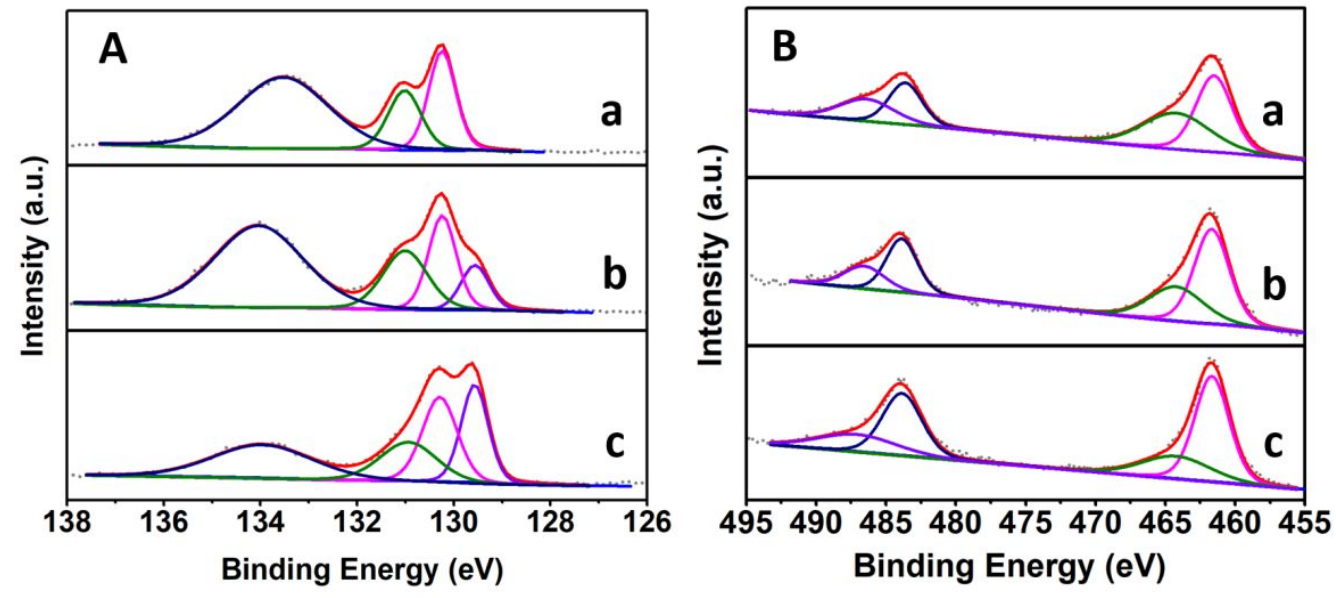

Figure S7.The XPS spectra of (A) P 2p, (B) Ru 3p for used catalysts. (a. $\left.\mathrm{Ru}_{2} \mathrm{P}, \mathrm{b} . \mathrm{RuP}, \mathrm{c} . \mathrm{RuP}_{2}\right)$
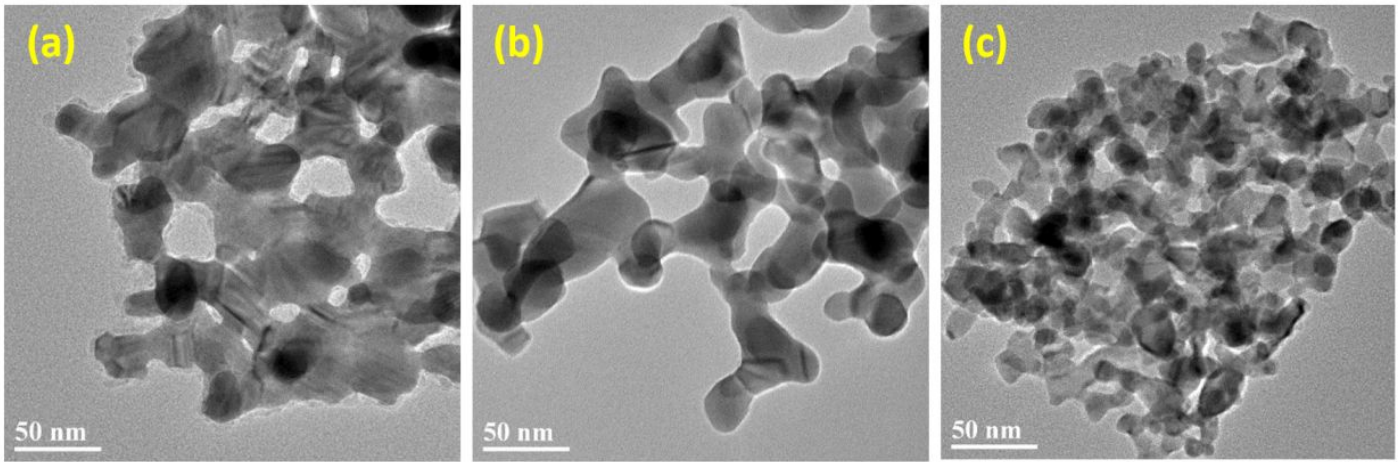

Figure S8. The HRTEM images used catalysts. (a. $\left.\mathrm{Ru}_{2} \mathrm{P}, \mathrm{b} . \mathrm{RuP}, \mathrm{c} . \mathrm{RuP}_{2}\right)$ 


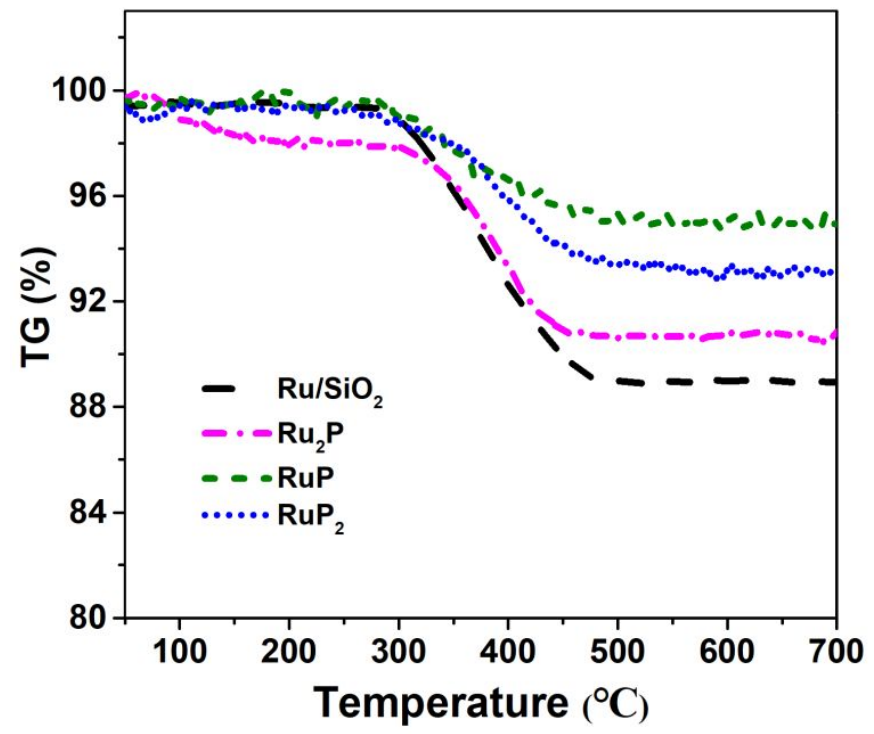

Figure S9. $T$ G curves under air atmosphere of $\mathrm{Ru}, \mathrm{Ru}_{2} \mathrm{P}, \mathrm{RuP}$ and $\mathrm{RuP} \mathrm{P}_{2}$ catalyst 